\title{
REPRESENTACCÕES SOCIAIS DE PROFISSIONAIS DE SAÚDE SOBRE A HIPERTENSÃO ARTERIAL: CONTRIBUIÇÕES PARA A ENFERMAGEM ${ }^{a}$
}

Social representations of health professionals about arterial hypertension: contributions to nursing

Representaciones sociales de profesionales de salud sobre la hipertensión arterial: contribuciones para la enfermería

\begin{abstract}
RESUMO
A Hipertensão Arterial, como grave problema de Saúde Pública, apresenta dificuldades de controle, destacando-se a dimensão psicossocial. 0 estudo objetivou apreender as representações sociais dos profissionais de saúde sobre a doença e as medidas de controle e explorar aspectos psicossociais relacionados aos conhecimentos, posicionamentos e comportamentos destes profissionais. Estudo exploratório, realizado em um Centro de Saúde, com 19 profissionais cujos dados foram coletados em três grupos focais e processados pelo software Alceste 4.8. As manifestações e descrições explicativas evidenciaram conhecimento dos profissionais sobre as dificuldades relacionadas às mudanças no estilo de vida das mulheres hipertensas e fatores de risco que contribuem para o surgimento da doença. 0 conhecimento destes aspectos traz contribuições por desvendar as questões subjetivas, peculiares da Hipertensão Arterial.
\end{abstract}

Palavras-chave: Saúde. Hipertensão. Cuidados de Enfermagem.

\begin{abstract}
Arterial Hypertension, as a serious public health problem, presents difficulties to be controlled, highlighting the psychosocial dimension. The study aimed to apprehend the social representations of health professionals about the disease and its control measures, and explore the psychosocial aspects related to the knowledge, attitudes and behaviors of these professionals. Exploratory study realized in a health center, with 19 professionals whose data were collected from three focal groups and processed by the software Alceste 4.8. The demonstrations and explanatory descriptions showed the knowledge of professionals about the difficulties related to changes in the lifestyles of women with hypertension, and risk factors that contribute to the emergence of the disease. The knowledge of these aspects contributes to unmask the subjective issues, peculiar to Arterial Hypertension.
\end{abstract}

Keywords: Health. Hypertension. Nursering cares.

\section{Resumen}

La Hipertensión Arterial, grave problema de Salud Pública, presenta dificultades de control destacándose la dimensión psicosocial. Así, este estudio tuvo como objetivos abarcar las representaciones sociales de los profesionales de salud sobre la Hipertensión Arterial y explorar aspectos psicosociales que influyen sus prácticas de control. Estudio exploratorio, de campo, llevado a cabo en Teresina-PI, con 19 profesionales, en un centro de salud. Los datos recolectados en tres grupos focales, organizados en "corpus" y procesados por el software Alceste 4.8. Las manifestaciones/descripciones explicativas evidenciaron el conocimiento de los profesionales sobre las dificultades relacionadas a los cambios en el estilo de vida de las hipertensas y factores de riesgo que contribuyen para el surgimiento de la enfermedad. El conocimiento de esos aspectos trae contribuciones por desvendar las cuestiones subjetivas peculiares a la Hipertensión Arterial.

Palabras clave: Salud. Hipertensión. Cuidados de enfermería.

'Mestre em Enfermagem. Profa . da NOVAFAPI. Enf. Da Fundação Municipal de Saúde de Teresina - PI. Brasil. Email: enoiasilva@hotmail.com, ${ }^{2}$ PósDoutora em Enfermagem Profa . Adjunta da UFPI e Coordenadora de Pesquisa e Pós-Graduação da NOVAFAPI, Teresina-PI. Brasil. Email: posgraduaçao@novafapi.com.br 


\section{INTRODUÇÃO}

Abordar a Hipertensão Arterial (HA) enquanto problema de Saúde Pública que afeta grande parte da população mundial implica a busca da compreensão das dificuldades de cunho psicossocial, econômico, biológico e cultural que envolve os seus portadores. Entender estas dificuldades torna-se relevante, principalmente ao articulá-las às condições de produção de conhecimento sobre a doença no imaginário social.

Este conhecimento possibilita a identificação de experiências vivenciadas pelos portadores, familiares e profissionais de saúde em relação à doença e às suas medidas de controle. Dentre seus agravos, salientam-se as doenças cardiovasculares e cerebrais como o Acidente Vascular Encefálico (AVE), uma das principais causas de morte originária da HA não controlada. ${ }^{1,2}$

As dificuldades de controle da HA estão relacionadas às características da doença, como o caráter assintomático, a evolução lenta, a cronicidade, que fazem com que não seja considerada doença ou algo que precise ser cuidado. Assim, os portadores não sentem necessidade de modificar os hábitos relacionados ao trabalho, ao meio social e à dinâmica familiar, até que surjam as primeiras complicações provocadas pela doença. $^{3}$

Quanto aos aspectos psicossociais, de modo geral, estão relacionados às crenças sobre a doença, à modificação dos hábitos e costumes necessários à convivência de seus portadores no âmbito sociofamiliar. Ressalta-se, ainda, que outros aspectos também são decisivos nesse processo de mudança, como a oferta e serviços de saúde à população, a condição socioeconômica e as desigualdades sociais, que agem coletivamente na saúde da população. ${ }^{4}$

$\mathrm{Na}$ busca desta dimensão, este estudo teve como objetivos apreender as representações sociais de profissionais de saúde que assistem mulheres portadoras de HA sobre a doença e as medidas de controle e explorar os aspectos psicossociais relacionados aos conhecimentos, posicionamentos e comportamentos capazes de influenciar as práticas desses sujeitos na orientação para o tratamento e controle da doença.

Neste contexto, é elevado número de portadores que ainda não responde satisfatoriamente às recomendações para o tratamento e controle da HA, mesmo que sejam priorizadas as ações educativas propostas pelo Programa Nacional de Educação e Controle da Hipertensão Arterial do Ministério da Saúde. Essas ações envolvem orientações para o autocuidado, para o tratamento medicamentoso e às mudanças no estilo de vida pertinentes ao controle dos fatores de risco que predispõem a doença e seus agravos. ${ }^{5}$

As orientações sobre a doença enfocadas pelos profissionais, na realidade desses serviços de saúde, estão parcialmente voltadas aos aspectos biológicos, epidemiológicos, econômicos, sendo pouco priorizados os aspectos sociopsicológicos e culturais. As ações educativas são desenvolvidas de forma autoritária e distanciadas da realidade social, deixando-se de lado as crenças, os sentimentos, a cultura, os hábitos alimentares dos portadores com relação a HA. ${ }^{6}$

0 tratamento e controle da $\mathrm{HA}$, na realidade do estudo, representam desafios às autoridades governamentais e aos próprios profissionais de saúde em face às dificuldades biológicas, psicossociais, econômicas e culturais que envolvem essas portadoras. Na dimensão da subjetividade, a investigação dessas dificuldades, segundo os profissionais de saúde, possibilita a apreensão de seus pensamentos, sentimentos e percepções, face às suas vivências, atitudes, valores e informações, segundo as experiências no processo de cuidar dos portadores da doença. ${ }^{6}$

Com base no exposto, supõe-se que a articulação entre a Hipertensão Arterial e as representações sociais dos profissionais de saúde sobre a doença e as suas medidas de controle poderão

ampliar o entendimento da construção psicossocial da doença no âmbito da adoção de medidas preventivas por esses profissionais e na compreensão de comportamentos dos portadores diante da doença. ${ }^{6}$

A relação estabelecida entre a HA e estes sujeitos encontrase permeada de conhecimentos advindos de seus diferentes contextos sócio-históricos, que se organizam em verdadeiros emaranhados de significados, permitindo a apreensão dos acontecimentos da vida diária, das características do meio ambiente e das informações que aí circulam sobre esta doença. Dessaforma, este estudo considera que os profissionais de saúde que assistem mulheres portadoras de Hipertensão Arterial acreditam que estas têm conhecimentos espontâneos sobre a doença, ou seja, um saber de senso comum através do qual se comunicam socialmente e se orientam no dia a dia rotineiro. ${ }^{6}$

Nesta perspectiva, a Teoria das Representações Sociais atende às expectativas de investigação e apreensão do conhecimento, posicionamentos, atitudes, sentimentos e comportamentos particulares desses sujeitos, bem como explora os aspectos subjetivos que possam influenciar no controle da doença, a partir do saber informal, estruturado socialmente e manifestado em suas condutas e comportamentos. ${ }^{6}$

As Representações Sociais são provenientes do julgamento do saber comum elaborado a partir dos fenômenos que emergem dos processos comunicacionais, os quais repercutem sobre as interações e as mudanças sociais, respondendo às expectativas de interpretação do fenômeno observado.?

Considerando que o entendimento dessa doença é permeado de subjetividade, sendo percebida de diferentes formas pelos seus portadores, espera-se que estes possuam representações divergentes, conturbadas e ambíguas. Isso porque estas são originadas de figuras, imagens, atos ou das experiências cotidianas, as quais os indivíduos atribuem um significado ou símbolo, que podem originar comportamentos ou atitudes. 
As experiências, vivências das pessoas com a doença, encontram-se inscritas em um contexto ativo, dinâmico, que participam da vida em coletividade, como prolongamento de seus comportamentos, tornando-os responsáveis pelo controle ou não da Hipertensão Arterial e, consequentemente, pela qualidade de suas vidas.

\section{METODOLOGIA}

Trata-se de um estudo exploratório, de campo, com emprego da Teoria das Representações Sociais. Este trabalho foi desenvolvido a partir de uma pesquisa mais ampla denominada "As representações sociais da hipertensão arterial", do Programa de Pós-graduação Mestrado em Enfermagem da Universidade Federal do Piauí-UFPI. Teve como cenário um centro de saúde da Estratégia Saúde da Família, com 19 profissionais de saúde que prestam assistência às mulheres cadastradas no Programa de Controle de Hipertensão Arterial.

Os sujeitos foram selecionados conforme critérios estabelecidos pela Resolução 196/96 do Conselho Nacional ${ }^{8}$ e assinaram o Termo de Consentimento Livre e Esclarecido, após autorização do Comitê de Ética e Pesquisa da Universidade Federal do Piauí, Parecer n0143/2004.

As informações foram coletadas pela técnica de grupo focal, em três sessões, com os profissionais de saúde, os quais foram orientados à discussão livre da seguinte questão (ou foco): A Hipertensão Arterial e suas medidas de controle. Estas discussões foram gravadas e, posteriormente, transcritas, lidas e relidas para melhor compreensão das falas destes profissionais. Tais informações foram organizadas num "corpus" e submetidas ao software Alceste $4.8^{\left({ }^{(9)}\right.}$ e posteriormente analisadas através da Classificação Hierárquica Descendente.

Este software auxilia na análise de dados textuais, com base nas leis de distribuição do vocabulário de falas e textos, possibilitando aos pesquisadores a análise de conteúdo deste tipo de matéria. ${ }^{10}$

Destaca-se, ainda, que este programa pode indicar as representações ou campos de imagens sobre um objeto, ou apenas aspectos de uma representação social, dependendo do conteúdo e da relação estabelecida entre esse objeto e o plano de pesquisa. ${ }^{8}$

\section{AS REPRESENTAÇÕES SOCIAIS DOS PROFISSIONAIS DE SAÚDE SOBRE A HIPERTENSÃO ARTERIAL}

0 software Alceste 4.8 produziu o dendograma das classes, com base na Classificação Hierárquica Descendente, sendo três delas tomadas como foco de análise e discussão deste estudo, para a evidência das representações sociais dos profissionais de saúde sobre a HA:

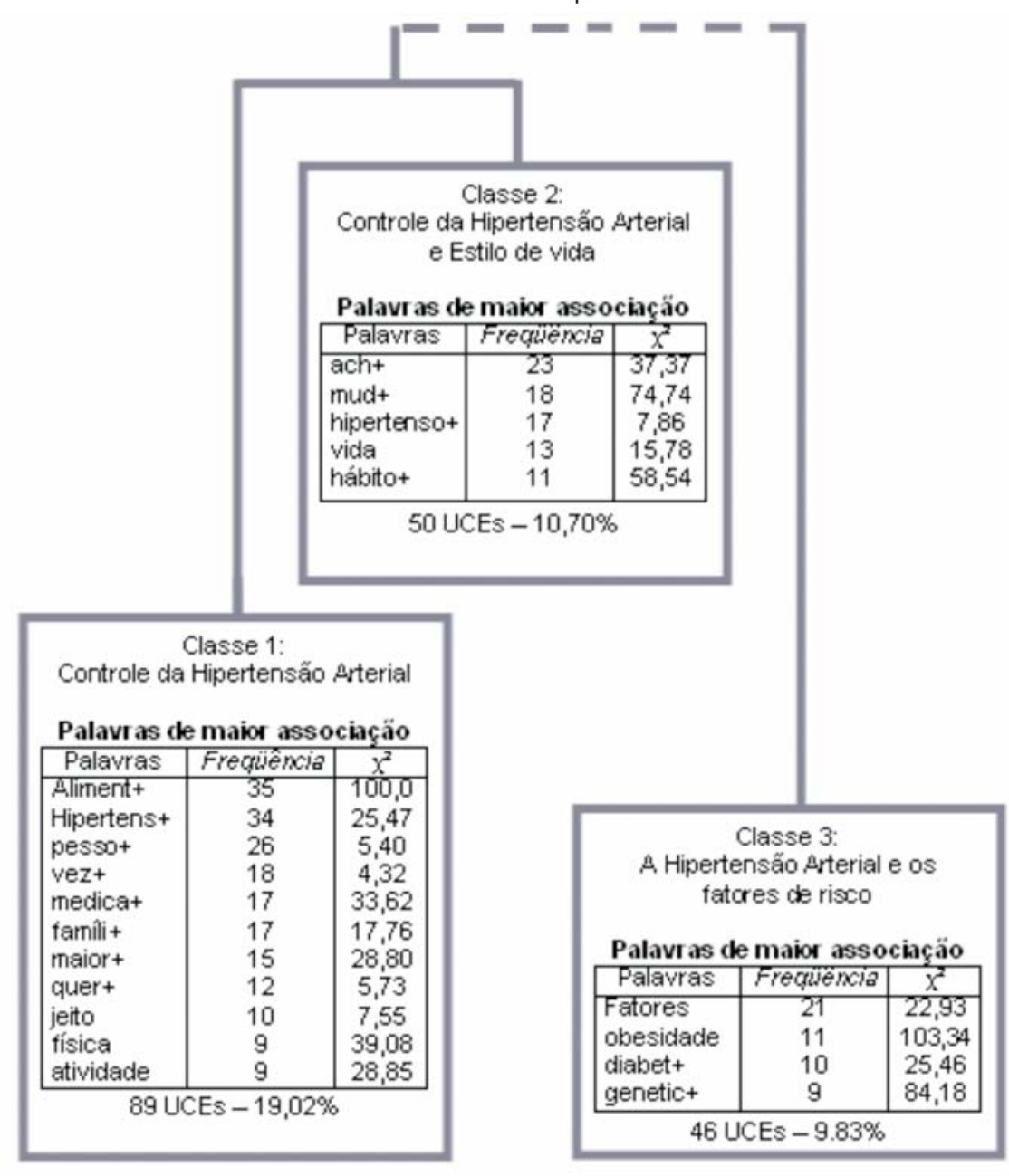


Classe 1 - Controle da Hipertensão Arterial - Traz evidências da presença de elementos sociocognitivos, afetivos e culturais das representações sociais desses sujeitos relativos aos posicionamentos: positivo, negativo e neutro, das mulheres portadoras de HA, diante das medidas de controle.

Os vocábulos da classe, em suas formas reduzidas: "aliment+", "física+", "médico+", "atividade+", maior+", hipertens+" e "famili+" selecionados pelos maiores valores de $\div^{2}$, correspondendo respectivamente: 100,0; 39,08; 33,62; 28,$85 ; 28,80 ; 25,47 ; 18,15$ e 17, 76, associados aos demais vocábulos em suas formas completas, nas UCEs, confirmam estas evidências e revelam a fonte de conhecimento do "senso comum" que orienta essas portadoras na vida prática, em seus ambientes sociofamiliares, relacionado à patologia.

Tais vocábulos apresentam as manifestações/descrição explicativas das dificuldades de controle da HA mencionadas pelos profissionais de saúde que apontam os conflitos das portadoras oriundos das necessidades desse controle, muitas vezes extensivas aos familiares e que assumem papel importante no insucesso da adesão às medidas:

\section{(...) também, outro ponto, é que a maioria das pessoas que têm hipertensão agravada é mais rebelde. Elas não querem seguir conselhos de uma pessoa mais jovem. (...) que elas não querem obedecer aos seus familiares.}

Estudos mostram fatores que exercem influências no comportamento de mulheres hipertensas, podendo relacionarse à própria doença, com suas características de cronicidade e inespecificidade: ao tratamento que envolve o uso constante de medicamentos; às questões institucionais associadas à distribuição de medicamentos e à dificuldade de acesso às ações de controle e, por fim, aos portadores, ressaltando-se os aspectos biológicos, hereditários, socioeconômicos e psicossociais, relacionados à doença. ${ }^{6}$

Ainda, como fatores geradores de conflitos à adesão das mulheres hipertensas às medidas de controle da hipertensão arterial, se destacam as mudanças nos hábitos alimentares especialmente quando estas convivem com familiares que não aderem a essas mudanças em função de suas próprias necessidades, que geralmente são outras. Assim, a falta de apoio dos familiares também concorre para que o controle da doença seja comprometido:

Eu acho que a pessoa quando descobre que é hipertensa há um conflito na família, porque ela tem que fazer uma dieta e o restante da familia não vai estar preocupado com sua dieta, uma dieta sem sal, com essas coisas (...).

Nesse aspecto, os estudos revelam que para a doença ser controlada é imprescindível a adesão ao tratamento, a qual é caracterizada pelo grau de cumprimento das medidas terapêuticas recomendadas, podendo ser ou não medicamentosas, objetivando a manutenção da pressão arterial em valores considerados normais com $\mathrm{PA}<140 / 90 \mathrm{mmHg} .{ }^{11}$

As dificuldades relativas ao poder econômico das mulheres portadoras de HA, para as mudanças necessárias ao controle da doença, ficam evidenciadas no conjunto de UCE provenientes de suas falas. Para elas, a não existência de recursos financeiros é condição para que não adquiram os alimentos recomendados:

Mas, muitas vezes, a comida é uma só, então, não vai mudar só o hábito do hipertenso, vai mudar o hábito alimentar da família. (...) realmente, como você vai passar uma dieta para quem só tem isso?

Outro fator importante relacionado aos hábitos alimentares é a indisponibilidade dos familiares para o preparo da alimentação, quando a maioria trabalha fora do lar, ficando o próprio "idoso" responsável por esse preparo:

(...) é a preocupação de que, também, a maioria dos hipertensos, principalmente, no caso dos idosos, é que vivem muito sós. As pessoas da família que convivem com eles trabalham durante o dia, então, nem cuidam da alimentação.

Quanto aos vícios, os profissionais de saúde apontam as dificuldades das mulheres portadoras de HA em abandonálos, em especial o tabagismo e o alcoolismo. Também a incorporação de atividades físicas são importantes na prevenção e tratamento da doença, representando desafios às autoridades sanitárias, aos profissionais de saúde, aos próprios portadores e familiares envolvidos na promoção de saúde. ${ }^{12}$.

Embora os profissionais de saúde considerem 0 abandono dos vícios pelas portadoras de HA uma conquista difícil, quando comparado ao abandonado de certos hábitos alimentares como a ingestão de alimentos com excesso de sal de cozinha e gorduras, torna-se mais fácil, considerando que a mudança nos hábitos alimentares às vezes implica também mudança de hábitos de outras pessoas do convívio familiar. Estas, pelo fato de não estarem doentes, não buscam compreender a importância de uma alimentação saudável, para a prevenção desta e de outras doenças:

(...) muitos vícios continuam os mesmos, principalmente, o fumo. É mais difícil tirar das pessoas, controlar. (...) a alimentação não é muito problema, o problema é controlar o peso (...). (...) acham que a maior dificuldade de ser portador de hipertensão está no convívio familiar, principalmente, na questão alimentar (...). 
Reforçando essas barreiras, as ameaças constantes das complicações da doença como o acidente vascular cerebral, "derrame cerebral", produzem insegurança, medo de invalidez, e até mesmo da morte. 0 medo da dependência dos familiares ou em situação oposta, que seria o agravamento das condiç̧ões econômicas quando as portadoras são as provedoras do próprio sustento e da família, traz angústia e sofrimento que pode contribuir para a negação da condição de doentes como forma de fugir da situação.

Neste contexto, o conhecimento da subjetividade dos sujeitos sobre a HA favorece a apreensão de pensamentos, sentimentos, percepções, representações, em face da vivência dos portadores e profissionais de saúde. Esses elementos se fazem presentes nas crenças, atitudes, valores e informações, segundo experiências subjetivas da comunicação entre estes sujeitos nos grupos aos quais pertencem. ${ }^{13}$.

(...) acho que é isso mesmo, em relação à hipertensão, daria para fazer um controle, usaria um medicamento até para manter um nível satisfatório e que interessa a toda clientela para evitar complicações mais sérias (...).

Outros vocábulos indicam posicionamentos neutros relacionados às características da doença, como a ausência de sintomatologia específica e o seu desenvolvimento gradual e silencioso. Estes posicionamentos concorrem para que as portadoras não deem à devida importância às medidas de controle recomendadas pelos profissionais, chegando mesmo a ignorar a doença e só procurarem atendimentos médicos nas situações de urgência determinadas pela elevação da pressão arterial. ${ }^{6}$

A questão da subjetividade fica muito presente nas falas das portadoras segundo a ótica dos profissionais de saúde, e possibilita a evidência das representações sociais como uma organização simbólica sobre a qual se desenvolvem as diferentes práticas e relações sociais em torno do controle da HA, por parte das portadoras, dos profissionais, da instituição e comunidade, pois representa uma produção subjetiva. Neste sentido, a utilização da Teoria das Representações Sociais, como suporte para interpretação do fenômeno desse estudo, realça o social que deixa de ser percebido em seu caráter objetivo e externo em relação aos sujeitos envolvidos nas diferentes práticas sociais.

As representações sociais, portanto, constituem uma forma de produção de conhecimento que assegura a estabilidade do "mundo", na qual as pessoas acreditam, o que representa um processo de produção subjetiva que garante a identidade e a segurança das pessoas: ${ }^{14}$

0 medo do que é estranho é profundamente arraigado. Isso se deve ao fato de que a ameaça de perder os marcos referenciais, de perder contato com o que propicia um sentido de continuidade de compreensão mútua, é uma ameaça insuportável.

Nesta perspectiva, as representações sociais dos sujeitos do estudo constituem a "realidade conhecida" à qual se atribui um valor de realidade como forma de preservar sua própria subjetividade. Portanto, esta teoria enfatiza a construção social da realidade em torno do controle da Hipertensão Arterial.

Classe 2 - Controle da Hipertensão Arterial e Estilo de Vida - aprofunda o conhecimento dos profissionais sobre as dificuldades de controle da HA relacionadas às mudanças em seu estilo de vida.

Os vocábulos mais frequentes, em suas formas reduzidas: "mud+", "hábito+", "ach+", "vida", "hipertenso+" e "cois+" e valores de $\div 2$ mais elevados, respectivamente: 74,74; 58,54; 37,37; 15,78; 7,86 e 4, 5, associados às formas completas nas UCEs da classe, trazem evidências de que a HA interfere na vida das portadoras, principalmente por não terem consciência de que é possível viverem com a doença sem perderem a qualidade de vida.

Estes elementos ressaltam as dificuldades inerentes ao processo de "mudança", sendo passíveis de conflitos e indagações, requerendo das mulheres portadoras uma gradativa adaptação e, por conseguinte, um trabalho profissional, no sentido de ajudá-las a superar as dificuldades oriundas desse processo:

(...) você não muda, é muito difícil porque a hipertensão não dói, não coça. Você não pega, você não vê, não é? E, (...) de um modo geral, você não sente nada, então é difícil você se conscientizar que você tem que mudar seus hábitos para favorecer (...).

Com relação ao tratamento anti-hipertensivo, o conteúdo da classe reitera as dificuldades das portadoras em aceitarem outras medidas terapêuticas associadas à medicamentosa, por não serem conscientes da doença em si e das possibilidades de controle a partir da incorporação da prática de exercícios físicos, da mudança nos hábitos alimentares e da redução do estresse: 6

(...) então, tudo é o remédio. (...) isso demonstra bem a falta de consciência sobre a doença e a possibilidade que têm para melhorar sua qualidade de vida, mesmo sendo portadores da doença. (...) tudo bem, você sabe de tudo, mas, a pessoa não tem condição de absorver (...).

A adesão às medidas de controle da HA é expressa pelo comportamento dos portadores em tomar medicamentos, 
aceitar a dieta orientada, comparecer às consultas médicas préagendadas e apresentar mudanças no estilo de vida. ${ }^{15-16}$.

0 tratamento anti-hipertensivo é imprescindível ao controle dos fatores de risco e na ausência, a doença pode resultar em agravos mais complexos. Assim, as representações sociais dos profissionais ressaltam suas descrenças nas medidas de controle da doença, considerando as dificuldades relacionadas à aprendizagem das portadoras diante das orientações sobre essas medidas.

As representações dos profissionais abordam os efeitos psicológicos positivos originários do processo de convivência das mulheres portadoras quando aceitam as mudanças no estilo de vida em função do controle da doença. Na experiência dos profissionais, a patologia controlada permite uma vida normal no âmbito sociofamiliar e de trabalho.

A classe revela que os profissionais têm conhecimentos sobre a fisiopatologia da doença hipertensiva e de suas medidas de controle. Reafirmam que, na condição de portadores da HA, também teriam dificuldades de adesão a essas medidas, pois suas características (assintomática, silenciosa, crônica, de evolução gradativa) não impõem limites aos portadores, conferindo-lhes uma falsa condição de saúde, até que sejam acometidas pelas complicações que colocam em risco suas vidas.

Os portadores de HA fazem associação da percepção da doença com o desenvolvimento de suas ações, principalmente àquelas que dizem respeito ao trabalho. 0 "adoecer" para as pessoas está relacionado a sintomas que comprometem seus a fazeres, o meio de subsistência, e pode ser entendido quando 0 corpo já não responde às exigências do convívio familiar e social..$^{17}$

Percebe-se, pois, que o número de portadores que não consegue manter os valores satisfatórios da pressão arterial, ainda que haja esforços por parte dos profissionais de saúde, indica a dificuldade de se controlar a doença, fato que contribui para acentuar os agravos e piorar a qualidade de vida, especialmente pelas sequelas e pelo aumento da permanência hospitalar. ${ }^{6}$

As representações sociais desses profissionais emergem de uma formação subjetiva das práticas em vigor na sociedade e na cultura. Os princípios de "relevância cultural" e de "espessura social" justificam a existência de representações, ou seja, o objeto de estudo se encontra implicado de forma consistente, em alguma prática do grupo relacionada ao sujeito e ao tema estudado, o que deve constituir um saber efetivamente praticado. Não deve ser apenas suposto, mas detectado em comportamentos e comunicaç̃es, que de fato ocorra sistematicamente.?

A partir dos anos de 1960, a Teoria das Representações Sociais vem sendo aplicada por vários autores em estudos de diferentes áreas de conhecimento, pois sua aplicação associada a metodologias que thes são apropriadas fornece o campo representacional de grupos sociais sobre diferentes objetos de estudo, ${ }^{18}$ importantes para a assistência à saúde.

Classe 3 - A Hipertensão Arterial e os Fatores de Risco - Reforça o conhecimento dos profissionais sobre as causas da
HA, com ênfase nos fatores genético-hereditários que estão fora do controle desses profissionais como nos problemas cardíacos.

As palavras em suas formas reduzidas: "obesidade", "genetic+", "diabet+", "fatores", selecionadas pelos valores de $\div 2$ mais elevados na classe, correspondendo respectivamente: 103,$34 ; 84,18 ; 25,46 ; 22,93 ; 20,70$ e 17,87 , quando associadas às suas formas completas, evidenciam seus conhecimentos sobre os fatores de risco da HA.

Os vocábulos da classe são indicativos de que os profissionais consideram os fatores genético-hereditários como os fatores de difícil controle e, por isso, longe do alcance do trabalho que realizam. Dessa forma, relacionam-se às dificuldades e à descrença dos profissionais no controle da doença:

Um caso que eu estou vendo dentro da minha familia é que não tem antecedente familiar, mas, que estão aparecendo os hipertensos e diabéticos. (...) carregam os genes com os problemas também.

Nesta classe, os vocábulos tratam dos fatores de risco associados ao estilo de vida das portadoras nos quais os profissionais destacam o estresse, o sedentarismo, hábitos alimentares, a obesidade, a diabetes, como relevantes para manutenção da epidemiologia da HA.

As dificuldades de controle da doença relacionam a adesão ou não a essas medidas e está na dependência das portadoras, fato que as responsabiliza pela problemática em questão:

(...) meu irmão com quarenta e poucos anos, com diabetes e com hipertensão associada à obesidade porque todo mundo era bem magro, bem magro mesmo, tudo magrinho, (...) e quando adultos estão todos com obesidade.

Quanto à vivência diária dos profissionais e das informações provenientes dos meios de comunicação (revistas e a televisão), as UCEs seguintes revelam suas posições quanto à adesão das mulheres portadoras $\mathrm{e}$ às medidas de controle relacionadas aos fatores de risco da HA:

Agora, tem uns casos, vi também na revista Veja que tem a obesidade mórbida, eles eram hipertensos, mas diminuíram o peso, voltaram ao peso normal, fizeram uma dieta, os exercícios e deixam de ser hipertensos.

Os profissionais de saúde demonstram suas manifestações/explicação quanto ao posicionamento 
positivo e negativo por eles assumidos, mostrando os efeitos psicológicos como as emoções a que essas mulheres portadoras são submetidas no dia-a-dia: o estresse e as preocupações como determinantes da elevação da pressão arterial.

Nesse contexto, ressaltam os aspectos sociais como os mais difíceis de serem controlados apesar de que estes aspectos, quando considerados, poderão contribuir também para a condição de normalidade da pressão arterial das portadoras.

Outro aspecto importante expresso na classe é o conhecimento dos profissionais de saúde e o das mulheres portadoras de HA, considerando suas fontes, sendo que as portadoras buscam o imaginário social para as explicações sobre o aparecimento e manutenção do quadro hipertensivo:

\section{(...) tem não, cura não, tem controle com a medicação, também do estresse, da alimentação, do peso, aí tem o controle, mas, curar mesmo, não tem não. E assim a literatura até mostra que junto aos fatores biológicos estão os sociais que são os mais difíceis até de serem controlados (...).}

Os profissionais de saúde, mesmo tendo conhecimento relativo à doença, buscam o conhecimento do senso comum para explicarem a relação da doença com seus fatores de risco. ${ }^{6}$. Evidenciam a função das representações sociais no processo de produção e circulação de conhecimentos que orienta e define os comportamentos e as condutas das portadoras e deles próprios, diante da doença hipertensiva.

\section{CONSIDERAÇÕES FINAIS}

0 estudo permitiu a evidência das diferentes concepções, atributos, posicionamentos e sentimentos dos profissionais de saúde, resgatando o saber do senso comum, sobre a HA. Ao mesmo tempo aproximou o saber científico, considerado do mundo intelectualizado, "quase" absoluto, desvelando aquilo que parecia não ser conhecimento essencial à compreensão dos aspectos subjetivos inerentes ao processo de se "adoecer" e "conviver", indefinidamente, com uma patologia como a HA.

0 conhecimento extraído das falas dos sujeitos facilitou a apreensão das representações sociais existentes nas manifestações/descrição/explicações que justificam seus posicionamentos, explorando os efeitos psicossociais expressos coletivamente, nos sentimentos desses sujeitos.

Em face do exposto, fica evidenciado que o conhecimento técnico, "reificado", dos profissionais de saúde é mais amplo quando comparado ao das mulheres portadoras que relacionam o não controle da doença apenas a fatores emocionais surgidos de suas relações sociofamiliares.
Os resultados mostram que, embora os profissionais de saúde desenvolvam ações educativas direcionadas às medidas de controle da HA, as portadoras demonstram não absorverem tais ensinamentos e agem orientadas pelo aprendizado da vida diária, a partir de suas experiências, ou seja, no conhecimento do "senso comum", veiculado pelo processo de comunicação que se estabelece em seus grupos de "pertença".

A instituição de saúde, aqui representadas por esses profissionais, carece de práticas articuladas às reais necessidades da população visto que estes desconhecem os aspectos psicossociais e culturais que podem influenciar 0 comportamento das portadoras perante as práticas de controle da Hipertensão Arterial.

\section{REFERÊNCIAS}

1. Ministério da Saúde (BR). Departamento de Atenção Básica. Norma Operacional de Assistência à Saúde - NOAS 01/2001. Brasília(DF); 2001.

2. Saraiva KLRO, Santos ZMSA, Landim FLP, Lima HP, Sena VL. 0 processo de viver do familiar cuidador na adesão do usuário hipertenso ao tratamento. Texto\&Contexto Enferm. 2007 jan/mar; (16)1: 63-70.

3. Santos ZMSA, Lima HP. Tecnologia educativa em saúde na prevenção da hipertensão arterial em trabalhadores: análise das mudanças no estilo de vida. Texto\&Contexto Enferm. 2008 jan/mar; 17(1): 90-7.

4. Teixeira ER, Silva JC, Lamas AR, Matos RM. 0 estilo de vida do cliente com a hipertensão arterial e o cuidado com a saúde. Esc Anna Nery. 2006 dez; 10(3): 378-84.

5. Ministério da Saúde (BR). Secretaria Nacional de Doenças CrônicoDegenerativas. Normas Técnicas para o Programa Nacional da Hipertensão Arterial (PNECHA). Brasilia(DF); 1988.

6. Silva MEDC. As representações sociais da hipertensão arterial [dissertação]. Teresina (PI): Universidade Federal do Piauí; 2006.

7. Moscovici S. A representação social da psicanálise. Rio de Janeiro (RJ): Zahar; 1978.

8. Ministério da Saúde (BR). Conselho Nacional de Saúde. Resolução n. 196/1996. Brasília(DF); 2003.

9. Reinert M. ALCESTE: analyse de donnes textuelles. Paris(FR): Societé Image; 1998.

10. Nascimento-Schulze CM, organizador. Dimensão da dor no câncer: reflexões sobre o cuidado interdisciplinar e um novo paradigma da saúde. São Paulo(SP): Probel; 1997.

11. Mano R. Considerações gerais sobre hipertensão arterial sistêmica. [on-line] 2002 [citado 2003 jul 10]. Disponível em: http:// uww.manuaisdecardiologia.med.br 
12. Pierin AMG, Strelec Junior M. 0 desafio do controle da hipertensão arterial e a adesão ao tratamento. In: Pierin AMG, organizador. Hipertensão arterial: uma proposta para o cuidar. Barueri(SP): Manole; 2004.

13. Péres DS, Magna JM, Viana LA. Portador de hipertensão arterial: atitudes, crenças, percepções, pensamentos e práticas. Rev Saude Publica 2003 out; 37(5): 635-42.

14. Moscovici S. Representações sociais: investigações em psicologia social. $4^{\text {a }}$ ed. Petrópolis(RJ): Vozes; 2003.

15. Sales CM, Tamaki EM. Adesão às medidas de controle da hipertensão arterial sistêmica: o comportamento do hipertenso. Cogitare Enferm. 2007 abr/jun; 12(2): 157-63.

16. Barbosa MRJ, Jorge MSB. Vivenciando a hipertensão arterial: construção de significados e suas implicações para a vida cotidiana. Rev Bras Enferm. 2000 out/dez; 53(4): 574-83.

17. Castro ME, Rolim MO. Conhecimento e esteriótipo de trabalhadores acerca da hipertensão. Esc Anna Nery. 2006 ago; 10(2):228-34.

18. Bursztyn I, Tura LFR. Avaliação em saúde e a teoria das representações sociais: notas para análise de possíveis interfaces. In: Moreira ASP, Jesuíno JC, organizadores. Representações sociais: teoria e prática. João Pessoa(PB): Universitária; 2003.

\section{NOTA}

a Dissertação de Mestrado do Programa de Mestrado de Enfermagem da Universidade Federal do Piauí. 